\title{
ASYMPTOTIC BEHAVIOUR OF THE ENERGY IN PARTIALLY VISCOELASTIC MATERIALS
}

\author{
BY \\ JAIME E. MUÑOZ RIVERA (National Laboratory for Scientific Computation, Department of \\ Research and Development, Rua Getulio Vargas 333, Quitandinha CEP 25651-070. Petrópollis, \\ $R J$, Brasil, and IM, Federal University of Rio de Janeiro)
}

AND

ALFONSO PERES SALVATIERRA (Universidad Nacional Mayor de San Marcos, Av. Venezuela $s / n$, Lima, Peru)

Abstract. In this paper we study models of materials consisting of an elastic part (without memory) and a viscoelastic part, where the dissipation given by the memory is effective. We show that the solutions of the corresponding partial viscoelastic model decay exponentially to zero, provided the relaxation function also decays exponentially, no matter how small is the viscoelastic part of the material

1. Introduction. Let us consider an $n$-dimensional body which in its reference configuration is homogeneous and occupies the open bounded set $\Omega \subset \mathbb{R}^{n}$ with smooth boundary $\Gamma$. Let $x \mapsto u(x, t)$ be the position of the material particle $x$ at time $t$. Then the viscoelastic equation of motion is given by

$$
\begin{gathered}
\left.\rho u_{t t}-\kappa \Delta u+\int_{0}^{\infty} g(s) \operatorname{div}\{a(x) \nabla u(\cdot, t-s)\} d s=f \quad \text { in } \Omega \times\right] 0, \infty[, \\
u(x, t)=0 \quad \text { on } \partial \Omega \times] 0, \infty[=\Gamma \times] 0, \infty[ \\
u(x, 0)=u_{0}(x), \quad u_{t}(x, 0)=u_{t}(x) \quad \text { in } \Omega
\end{gathered}
$$

where $\rho$ is the mass density function, $g$ is the relaxation function and $f$ denotes the body force. Here we are mainly interested in the asymptotic behaviour of the solution $u$ when $t$ tends to infinity. Note that the above model is dissipative, and the dissipation is given by the memory term, where $a \geq 0$. The memory is effective only in a part of the body $\Omega$ where $a>0$.

Received February 21, 2000.

2000 Mathematics Subject Classification. Primary 35B40, 35L05, 35L70.

Key words and phrases. Viscoelasticity, exponential decay, localized damping, materials with memory. Supported by a grant 305406/88-4 of CNPq-BRASIL. 
Concerning the exponential stability of dissipative systems, it is well known by now that the solution of the wave equation with frictional damping

$$
\begin{gathered}
\left.u_{t t}-\Delta u+a(x) u_{t}=0 \quad \text { in } \Omega \times\right] 0, \infty[, \\
u(x, t)=0 \quad \text { on } \Gamma \times] 0, \infty[
\end{gathered}
$$

decays exponentially to zero as time goes to infinity provided $a(x) \geq a_{0}>0$ a.e. in $\Omega$. In this case, since $a(x)$ is a positive function, the dissipative effect is working in the whole domain $\Omega$. In this direction we may ask whether the damping term $a(x) u_{t}$ continues to be effective when the function $a$ satisfies only $a(x) \geq 0$. That is, suppose that the function $a(x)$ vanishes outside an open subset $\omega$ of $\Omega$. Is the dissipative term $a(x) u_{t}$ strong enough to produce the exponential decay of the solution? Under what conditions on $\omega$ may we expect a uniform rate of decay for the solution? A first answer to these questions was given in [6], [14], [18], [27]. In those works the authors proved that the solution of the wave equation with "local" damping decays exponentially to zero provided $\omega$ is a neighborhood of the boundary $\Gamma$. This means that a dissipation mechanism that is effective over a strategic part of the material is strong enough to produce exponential rate of decay of the total energy. Such results are connected with Control Theory. It was proved by Lions [13] that the solution of the wave equation can be controlled acting only in a neighborhood of the boundary. Roughly speaking, any time that we can control the solution acting on a part of the material, it is possible to introduce a dissipative mechanism at the same place producing uniform decay in time of the energy. The methods in Bardos et al. [1] allow us to give a necessary and sufficient condition for the uniform decay of the solutions of the dissipative wave equation. Namely, this condition requests that every ray of geometric optics that propagates in $\Omega$ and is reflected on the boundary enters the region $a>0$ in a uniform time. In fact, the necessity of this condition is due to the construction of Ralston [22, 23] of the Gaussian beam solutions of the wave equation. Other examples of localized damping are given by introducing dissipative boundary conditions acting in a part of the boundary. In this direction there is an extensive literature; see for example [4], [5], [6], [7], [8], [10], [11], [12], [17], [19], [20], [21], [24], [26], [28] among others.

In this paper we also consider locally distributed dissipation; but this dissipation does not appear by the introduction of any artificial mechanism. On the contrary, it arises because of the mixed structure of the material. That is, we consider a body consisting of an elastic and a viscoelastic part. So, the dissipation is due to the memory effect which works only over a portion of the material. The one-dimensional case is represented in Fig. 1, but our analysis applies without restriction on the dimension.

Denoting by $\sigma$ the stress and by * the convolution product $g * f=\int_{0}^{t} g(t-\tau) f(\tau) d \tau$, the constitutive law we use in this paper is given by

$$
\sigma=\kappa \nabla u+a(x) g * \nabla u
$$

so that the corresponding motion equation for $\kappa=1$ may be written as

$$
u_{t t}-\Delta u+\int_{0}^{t} g(t-\tau) \operatorname{div}\{a(x) \nabla u\} d \tau=0 \quad \text { in } \Omega \times 0, \infty[
$$




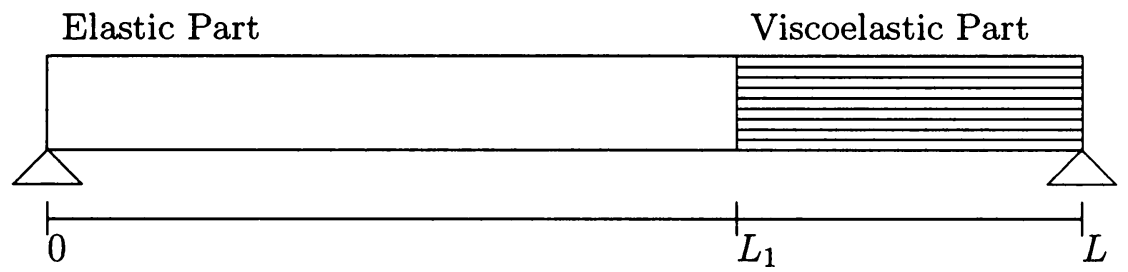

FIG. 1

with initial data

$$
u(x, 0)=u_{0}(x), \quad u_{t}(x, 0)=u_{1}(x) \quad \text { in } \Omega,
$$

and Dirichlet's boundary condition

$$
u(x, t)=0 \quad \text { on } \Gamma \times] 0, \infty[.
$$

For materials with memory the stress depends not only on the present values but also on the entire temporal history of the motion. Therefore, we have also to prescribe the history of $u$ prior to 0 as the initial data. Here we assume that $u$ vanishes identically for $t<0$, that is,

$$
u(x, t)=0, \quad \text { for } t<0 .
$$

We do not assume that $u\left(\cdot, 0^{+}\right)=u\left(\cdot, 0^{-}\right)$or $u_{t}\left(\cdot, 0^{+}\right)=u_{t}\left(\cdot, 0^{-}\right)$.

Let $x \mapsto a(x)$ be a nonnegative $C^{2}$-function defined over $\Omega$ and let us denote by $\omega_{\varepsilon}$ the set

$$
\omega_{\varepsilon}=\left(\bigcup_{x \in \Gamma_{0}} B_{\varepsilon}(x)\right) \cap \Omega,
$$

where $B_{\varepsilon}(x)=\left\{x \in \mathbb{R}^{n} ;\|x\| \leq \varepsilon\right\}$ and $\Gamma_{0}$ is given by

$$
\Gamma_{0}=\left\{x \in \Gamma ;\left(x-x_{0}\right) \cdot \nu \geq 0\right\},
$$

where $\nu$ is the unitary external normal defined over $\Gamma$ and $x_{0}$ is any point of $\mathbb{R}^{n}$. For the one-dimensional case we have that $\left.\omega_{\varepsilon}=\right] L-\varepsilon, L$. The hypotheses we use on $a$ are the following:

$$
\begin{gathered}
x \mapsto a(x) \in C^{2}(\bar{\Omega}) ; \quad a(x)= \begin{cases}0 & \text { on } \Omega \backslash \omega_{\varepsilon}, \\
1 & \text { on } \omega_{\varepsilon / 2},\end{cases} \\
|\nabla a(x)|^{2} \leq c|a(x)| .
\end{gathered}
$$

We can build a function $a$ satisfying condition (1.5) by taking $a=b^{2}$, with $b$ as in (1.4). In Fig. $2, \Omega$ is a rectangle and the set $\omega_{\varepsilon}$ denotes the viscoelastic part of the body. Note that $\omega_{\varepsilon}$ is behind the part that an observer can see when situated on $x_{0}$. This is a particular case in which the geometric requirements of Bardos et al. [1] and Ralston $[22,23]$ are satisfied.

Let us mention some other papers related to the problems we address. Dafermos in [2] proved that the solution to a viscoelastic system goes to zero as time goes to infinity, but without giving the explicit rate of decay. Lagnese in [9] considered the linear 


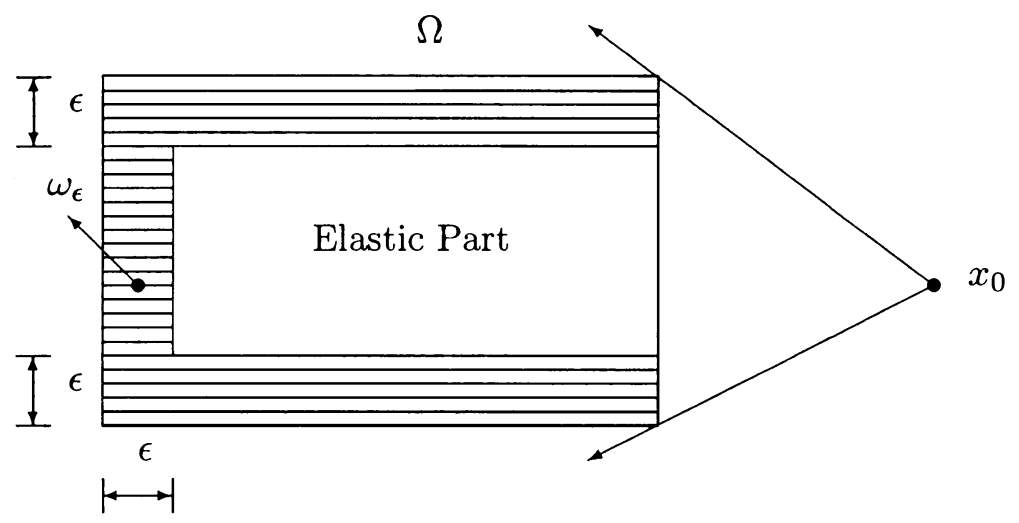

FI( .2

viscoelastic plate equation, obtaining uniform rates of decay but introducing additional damping terms acting on the boundary. Uniform rates of decay for the solutions of a linear viscoelastic system with memory were obtained recently by M. Rivera et al [15]. Unfortunately, the methods used to achieve uniform rates of decay in those works are based on second-order estimates, which are time-dependent in our problem. Thus, the methods that have been used for establishing uniform rates of decay fail in the case of a partially viscoelastic equation. Therefore, a new asymptotic technique has to be devised. For nonlinear models see [16].

The aim of this paper is to show that in the geometrical setting above, the energy decays exponentially provided the kernel $g$ also decays exponentially. More specifically, we assume that $g$ satisfies

$$
\begin{gathered}
g \in C^{3}(] 0, \infty[) . \quad \\
g(t)>0, \quad\left|g^{\prime \prime}(t)\right| \leq c g(t), \quad\left|g^{\prime \prime \prime}(t)\right| \leq c g(t), \\
-\kappa_{0} g(t) \leq g^{\prime}(t) \leq \kappa_{1} g(t), \\
\alpha:=1-\int_{0}^{\infty} g(\tau) d \tau<1 .
\end{gathered}
$$

To facilitate our analysis, we introduce the following binary operators:

$$
\begin{aligned}
g \square \nabla u & =\int_{0}^{t} g(t-\tau) \int_{\Omega_{2}} a(x)|\nabla u(x, t)-\nabla u(x, \tau)|^{2} d x d \tau, \\
g \square u & =\int_{0}^{t} g(t-\tau) \int_{\Omega} a(x)|u(x, t)-u(x, \tau)|^{2} d x d \tau .
\end{aligned}
$$

Under the above conditions the main result of this paper is given by

THEOREM 1.1. Under the above assumptions on $\Omega, \omega, a$, and with the kernel $g$ satisfying (1.6)-(1.8), the weak solution of the viscoelastic equation (1.1)-(1.3) decays exponentially as time goes to infinity. That is, there exist positive constants $c$ and $\gamma$ that do not depend on the initial data such that

$$
E(t) \leq c E(0) e^{-\gamma t}
$$


where by $E(t)$ we are denoting the first-order energy

$$
E(t)=\frac{1}{2} \int_{\Omega}\left|u_{t}\right|^{2}+\left\{1-a(x) \int_{0}^{t} g(\tau) d \tau\right\}|\nabla u|^{2} d x+\frac{1}{2} g \square \nabla u .
$$

The method we use here is based on the construction of a functional $\mathcal{L}$ for which an inequality of the form

$$
\frac{d}{d t} \mathcal{L}(t) \leq-c \mathcal{L}(t)
$$

holds, with $c>0$. To construct such a functional $\mathcal{L}$ we start from the energy identity. Then, we look for other functions whose derivatives introduce negative terms such as: $-\int a(x)\left|u_{t}\right|^{2}-\int a(x)|\nabla u|^{2}$, etc. until we are able to construct the whole energy in the right-hand side of the energy identity. Finally, we take $\mathcal{L}$ as the summation of such functions. Unfortunately, the above process also introduces terms without definite sign. To overcome this difficulty, we introduce a new multiplier, which allows us to get the appropriate estimates. Finally, we choose carefully the coefficients of each term of $\mathcal{L}$, such that the resulting summation satisfies the required inequality.

The remaining part of this article is organized as follows. In Sec. 2, we establish the existence and regularity result to equations (1.1)-(1.3) as well as the energy identity. Section 3 deals with the regularity of the convolution term. Finally, in Sec. 4 we show the exponential decay of the solution of the equations (1.1)-(1.3).

2. Existence results and preliminaries. Our starting point is given by the following lemma.

Lemma 2.1. For any $v \in C^{1}\left(0, T ; H^{1}(0, L)\right)$ we get

$$
\begin{aligned}
\int_{\Omega} \int_{0}^{t} g(t-\tau) a(x) \nabla v d \tau \cdot \nabla v_{t} d x= & -\frac{1}{2} g(t) \int_{\Omega} a(x)|\nabla v|^{2} d x+\frac{1}{2} g^{\prime} \square \nabla v \\
& -\frac{1}{2} \frac{d}{d t}\left\{g \square \nabla v-\left(\int_{0}^{t} g d \tau\right) \int_{\Omega} a(x)|\nabla v|^{2} d x\right\} \\
\int_{\Omega} \int_{0}^{t} g(t-\tau) a(x) v d \tau \cdot v_{t} d x= & -\frac{1}{2} g(t) \int_{\Omega} a(x)|v|^{2} d x+\frac{1}{2} g^{\prime} \square v \\
& -\frac{1}{2} \frac{d}{d t}\left\{g \square v-\left(\int_{0}^{t} g d \tau\right) \int_{\Omega} a(x)|v|^{2} d x\right\} .
\end{aligned}
$$

Proof. It is easy to see that

$$
\begin{aligned}
\frac{d}{d t}\{g \square \nabla v\}= & g^{\prime} \square \nabla v-2 \int_{\Omega} \int_{0}^{t} g(t-\tau) a(x) \nabla v(\tau) d \tau \cdot \nabla v_{t}(x, t) d \tau d x \\
& +2 \int_{0}^{t} g(t-\tau) d \tau \int_{\Omega} a(x) \nabla v \nabla v_{t} d x \\
= & g^{\prime} \square \nabla v-2 \int_{\Omega} \int_{0}^{t} g(t-\tau) a(x) \nabla v(\tau) d \tau \cdot \nabla v_{t} d x \\
& +\frac{d}{d t}\left\{\int_{0}^{t} g(\tau) d \tau \int_{\Omega} a(x)|\nabla v|^{2} d x\right\}-g(t) \int_{\Omega} a(x)|\nabla v|^{2} d x
\end{aligned}
$$


This shows our result. The proof of the other identity is similar.

It is not difficult to show that there exists only one solution to equations (1.1)-(1.3). We summarize the existence result in the following theorem.

Theorem 2.1. Let us suppose that $g$ is a $C^{0}$-function and that the initial data satisfies

$$
\left(u_{0}, u_{1}\right) \in H_{0}^{1}(\Omega) \times L^{2}(\Omega) .
$$

Then there exists only one weak solution $u$ to equations (1.1)-(1.3) with the following regularity:

$$
u \in L^{\infty}\left(0, \infty ; H_{0}^{1}(\Omega)\right), \quad u_{t} \in L^{\infty}\left(0, \infty ; L^{2}(\Omega)\right) .
$$

In addition, if $g \in C^{1}$ and

$$
\left(u_{0}, u_{1}\right) \in H^{2}(\Omega) \cap H_{0}^{1}(\Omega) \times H_{0}^{1}(\Omega),
$$

then there exists only one strong solution $u$ of equations (1.1)-(1.3) satisfying

$$
u \in C^{2-i}\left(0, \infty ; H_{0}^{1}(\Omega) \cap H^{i}(\Omega)\right), \quad i=1,2, \quad u \in C^{2}\left(0, \infty ; L^{2}(\Omega)\right) .
$$

The dissipative property of the viscoelastic equation is summarized in the following lemma.

LEMMA 2.2. Any strong solution of (1.1)-(1.3) satisfies

$$
\frac{d}{d t} E(t)=\frac{1}{2} g^{\prime} \square \nabla u-\frac{1}{2} g(t) \int_{\Omega} a(x)|\nabla u|^{2} d x .
$$

Proof. Multiplying Eq. (1.1) by $u_{t}$ and integrating over $\Omega$ yields

$$
\frac{d}{d t} \int_{\Omega}\left(\left|u_{t}\right|^{2}+|\nabla u|^{2}\right) d x=\int_{\Omega} a(x) g * \nabla u \cdot \nabla u_{t} d x .
$$

From Lemma 2.1 our conclusion follows.

Lemma 2.2 tells us that the dissipation given by the memory term is effective only on the support of the function $a$. We will show in Sec. 4 that such dissipation is enough to produce the exponential decay of the energy, as time goes to infinity.

3. Regularity of the convolution. Let us denote by $\|\cdot\|_{C^{0}}$ the norm in $C^{0}(\bar{\Omega})$. The following lemma will play an important role in the sequel.

LEMMA 3.1. Let us suppose that $g$ is a positive function satisfying condition (1.8), $a \in$ $C^{0}(\bar{\Omega})$ is such that $\|a\|_{C^{0}} \leq 1$ and finally let us take $f \in L^{p}\left(0, T ; L^{2}(\Omega)\right)$ with $1 \leq p<\infty$. Under these conditions we have that there exists only one solution $v$ of Volterra's equation

$$
\left.v(x, t)-\int_{0}^{t} g(t-\tau) a(x) v(\cdot, \tau)=f(x, t) \quad \text { a.e. }(x, t) \in \Omega \times\right] 0, T[
$$

satisfying

$$
v \in L^{p}\left(0, T ; L^{2}(\Omega)\right)
$$


Besides, there exists a positive constant $c$ independent of $T$ such that

$$
\|v\|_{L^{p}\left(0, T ; L^{2}\right)} \leq c\|f\|_{L^{p}\left(0, T ; L^{2}\right)} .
$$

Proof. We will use Picard's method. Let us denote by

$$
v_{0}:=f, \quad v_{-1}=0
$$

and consider the iterative equation

$$
v_{\mu}(x, t)=\int_{0}^{t} g(t-\tau) a(x) v_{\mu-1}(\cdot, \tau) d \tau+f(x, t) .
$$

Denoting by

$$
w_{\mu}=v_{\mu}-v_{\mu-1}
$$

we have that

$$
w_{\mu}(x, t)=\int_{0}^{t} g(t-\tau) a(x) w_{\mu-1}(\cdot, \tau) d \tau,
$$

from which it follows that

$$
\left\|w_{\mu}(t)\right\|_{L^{2}} \leq \int_{0}^{t} g(t-\tau)\|a\|_{C^{0}}\left\|w_{\mu-1}(\cdot, \tau)\right\|_{L^{2}} d \tau .
$$

Using Young's inequality we get

$$
\left.\left\|w_{\mu}\right\|_{L^{p}\left(0, T ; L^{2}\right)} \leq\left(\int_{0}^{T} g(\tau) d \tau\right)\right)\|a\|_{C^{0}}\left\|w_{\mu-1}\right\|_{L^{p}\left(0, T ; L^{2}\right)},
$$

from which it follows that

$$
\begin{aligned}
\left\|w_{\mu}\right\|_{L^{p}\left(0, T ; L^{2}\right)} & \leq\left[\left(\int_{0}^{T} g d \tau\right)\|a\|_{C^{0}}\right]^{2}\left\|w_{\mu-2}\right\|_{L^{p}\left(0, T ; L^{2}\right)} \\
& \leq\left[\left(\int_{0}^{T} g d \tau\right)\|a\|_{C^{0}}\right]^{\mu}\left\|w_{0}\right\|_{L^{p}\left(0, T ; L^{2}\right)} \\
& \leq\left[\left(\int_{0}^{T} g d \tau\right)\|a\|_{C^{0}}\right]^{\mu}\|f\|_{L^{p}\left(0, T ; L^{2}\right)} .
\end{aligned}
$$

Since $\left(\int_{0}^{T} g d \tau\right)\|a\|_{C^{0}}<1$, it follows that

$$
\sum_{\mu=1}^{\infty}\left\|w_{\mu}\right\|_{L^{p}\left(0, T ; L^{2}\right)} \leq \frac{\|f\|_{L^{p}\left(0, T ; L^{2}\right)}}{1-\int_{0}^{T} g d \tau\|a\|_{C^{0}}} \leq \frac{\|f\|_{L^{p}\left(0, T ; L^{2}\right)}}{1-(1-\alpha)\|a\|_{C^{0}}},
$$

where $\alpha$ was defined in (1.8). Recalling that

$$
v_{\mu}-v_{0}=\sum_{i=1}^{\mu} w_{i}
$$

we conclude that the sequence $v_{\mu}$ is convergent. So, there exists a function $v \in$ $L^{p}\left(0, T ; L^{2}(\Omega)\right)$ for which we have

$$
v_{\mu} \rightarrow v \quad \text { strong in } L^{p}\left(0, T ; L^{2}(\Omega)\right) .
$$


Besides, we have that

$$
\|v\|_{L^{p}\left(0, T: L^{2}\right)} \leq \underbrace{\left\{\frac{1}{1-(1-\alpha)\|a\|_{C^{0}}}+1\right\}}_{:=C}\|f\|_{L^{p}\left(0, T: L^{2}\right)},
$$

where $C$ is a positive constant that does not depend on $T$. To show the uniqueness, let us suppose that there exists another solution $\bar{v}$. Denoting by $V=v-\bar{v}$, we have that

$$
V=\int_{0}^{t} g(t-\tau) a(x) V(x, \tau) d \tau .
$$

In particular,

$$
|V| \leq \int_{0}^{t} g(t-\tau)|a(x) V(x, \tau)| d \tau .
$$

From Gronwall's inequality we conclude that $V=0$. The proof is now complete.

In the next lemma we will show that integration in time is equivalent to removing spatial derivatives.

LEMmA 3.2. Let us suppose that $0 \leq a(x) \leq 1$ satisfies conditions (1.6)-(1.8) and that $g$ is a positive function satisfying (1.8). If $u$ is a weak solution of (1.1)-(1.3) satisfying

$$
u_{t} \in L^{\infty}\left(0, T ; L^{2}(\Omega)\right), \quad u \in L^{\infty}\left(0, T ; H_{0}^{1}(\Omega)\right),
$$

then we have that

$$
g * u \in L^{2}\left(0, T ; H^{2}(\Omega)\right)
$$

and

$$
\|g * u\|_{L^{2}\left(0, T ; H^{2}\right)} \leq C \int_{0}^{T} E(t) d t+C E(0),
$$

where $C$ is a positive constant independent of $T$.

Proof. Applying convolution to Eq. (1.1), we have

$$
-\Delta g * u+g * g * \operatorname{div}\{a(x) \nabla u\}=-g * u_{t t} .
$$

Performing an integration by parts over $] 0, t$, we get

$$
g * u_{t t}=g(0) u_{t}-g(t) u_{t}(\cdot, 0)+\int_{0}^{t} g^{\prime}(t-\tau) u_{t} d \tau:=-F
$$

From the hypotheses we conclude that $F \in L^{2}\left(0, T ; L^{2}(\Omega)\right)$. Denoting by $v=g * \Delta u$, we have that

$$
-v+a(x) g * v=G,
$$

where

$$
G=F-g * \nabla a(x) \cdot \nabla u .
$$

Applying Lemma 3.1 for $p=2$ and since $\|a\|_{C^{0}} \leq 1$ we conclude that

$$
v \in L^{2}\left(0, T ; L^{2}(\Omega)\right) \text {. }
$$


Since

$$
\begin{aligned}
\int_{0}^{T} \int_{\Omega}|F|^{2} d x & \leq 2 g(0)^{2} \int_{0}^{T} \int_{\Omega}\left|u_{t}\right|^{2} d x d t \\
& +\int_{0}^{T} g(t) \int_{\Omega}\left|u_{1}\right|^{2} d x d t+\underbrace{\int_{0}^{T} \int_{\Omega}\left|g^{\prime} * u_{t}\right|^{2} d x d t}_{\leq \int_{0}^{T}\left|g^{\prime}\right| d t \int_{0}^{T} \int_{\Omega}\left|u_{t}\right|^{2} d x}
\end{aligned}
$$

we have that

$$
\begin{aligned}
\|v\|_{L^{2}\left(0, T ; L^{2}(\Omega)\right)} & \leq c\|G\|_{L^{2}\left(0, T ; L^{2}(\Omega)\right)} \\
& \leq C \int_{0}^{T} E(t) d t+C \int_{0}^{T} g(t) d t E(0),
\end{aligned}
$$

which implies that $g * \Delta u \in L^{2}\left(0, T ; L^{2}(\Omega)\right)$, from which we obtain

$$
g * u \in L^{2}\left(0, T ; H^{2}(\Omega)\right) .
$$

Finally, using elliptic regularity, inequality (3.1) follows. The proof is now complete.

4. Exponential decay. To show the exponential decay of the solution let us introduce the following functional:

$$
\begin{aligned}
I(t):= & \int_{\Omega} a(x)\left\{u_{t}(g * u)_{t}-\frac{1}{2} g(0)|u|^{2}-\int_{0}^{t} g d \tau|u|^{2} d x\right\} d x \\
& -\frac{1}{2} g^{\prime \prime} \square u+\frac{1}{2} \int_{\Omega} a^{2}(x)|g * \nabla u|^{2} d x .
\end{aligned}
$$

In these conditions we have

LEMma 4.1. Under the above conditions and for $g \in C^{3}$, satisfying conditions (1.6)(1.8), we have that for any $\delta>0$ there exists $C_{\delta}$ satisfying

$$
\begin{aligned}
& \frac{d}{d t} I(t) \leq-g(0) \int_{\Omega} a(x)\left|u_{t}\right|^{2} d x+\delta \int_{\Omega} a(x)|\nabla u|^{2} d x \\
& \quad+C_{\delta} g \square \nabla u+C_{\delta} g(t) \int_{\Omega} a(x)|\nabla u|^{2} d x \\
& \quad+C_{\delta} \int_{\omega_{\varepsilon}} \int_{0}^{t} g(t-\tau)|u(x, t)-u(x, \tau)|^{2} d x d t+C_{\delta} \int_{\omega_{\varepsilon}}|u(x, t)|^{2} d x .
\end{aligned}
$$

Here $C_{\delta} \rightarrow \infty$ when $\delta \rightarrow 0$.

Proof. Multiplying Eq. (1.1) by $a(x)(g * u)_{t}$ we get

$$
\underbrace{\int_{\Omega} u_{t t} a(x)(g * u)_{t} d x}_{:=I_{1}}-\underbrace{\int_{\Omega} \Delta u a(x)(g * u)_{t} d x}_{:=I_{2}}-\underbrace{\int_{\Omega} a(x) g * \nabla u \cdot \nabla\left\{a(x)(g * u)_{t}\right\} d x}_{:=I_{3}}=0
$$


from which we have

$$
\begin{aligned}
I_{1}= & \frac{d}{d t} \int_{\Omega} u_{t} a(x)(g * u)_{t} d x-\int_{\Omega} u_{t} a(x)(g * u)_{t t} d x \\
= & \frac{d}{d t} \int_{\Omega} u_{t} a(x)(g * u)_{t} d x-\int_{\Omega} u_{t} a(x)\left(g(0) u+g^{\prime} * u\right)_{t} d x \\
= & \frac{d}{d t} \int_{\Omega} u_{t} a(x)(g * u)_{t} d x-g(0) \int_{\Omega} a(x)\left|u_{t}\right|^{2} d x \\
& -\int_{\Omega} a(x) u_{t}\left\{g^{\prime}(0) u+g^{\prime \prime} * u\right\} d x \\
= & \frac{d}{d t} \int_{\Omega} u_{t} a(x)(g * u)_{t} d x-g(0) \int_{\Omega} a(x)\left|u_{t}\right|^{2} d x-\frac{g^{\prime}(0)}{2} \frac{d}{d t} \int_{\Omega} a(x)|u|^{2} d x \\
& -\int_{\Omega} a(x) u_{t} g^{\prime \prime} * u d x
\end{aligned}
$$

Using Lemma 2.1 we get that

$$
\begin{aligned}
\int_{\Omega} a(x) u_{t} g^{\prime \prime} * u d x= & \frac{1}{2} g^{\prime \prime \prime} \square u-\frac{1}{2} \int_{\Omega} a(x)|u|^{2} d x \\
& -\frac{1}{2} \frac{d}{d t}\left\{g^{\prime \prime} \square u-\int_{0}^{t} g d \tau \int_{\Omega} a(x)|u|^{2} d x\right\}
\end{aligned}
$$

from which it follows that

$$
I_{1}(t)=\frac{d}{d t} I_{0}(t)-g(0) \int_{\Omega} a(x)\left|u_{t}\right|^{2} d x+\frac{1}{2} g^{\prime \prime}(t) \int_{\Omega} a(x)|u|^{2} d x-\frac{1}{2} g^{\prime \prime \prime} \square u,
$$

where by $I_{0}$ we are denoting

$$
I_{0}=\int_{\Omega} a(x)\left\{u_{t}(g * u)_{t}-\frac{g^{\prime}(0)}{2}|u|^{2}\right\} d x-\frac{1}{2} g^{\prime \prime} \square u-\int_{0}^{t} g d \tau \int_{\Omega} a(x)|u|^{2} d x .
$$

On the other hand,

$$
\begin{aligned}
I_{2}= & -\int_{\Omega} a(x) \Delta u\left\{g(0) u+g^{\prime} * u\right\} d x \\
= & \int_{\Omega} \nabla u \cdot \nabla\left\{a(x) g(0) u+a(x) g^{\prime} * u\right\} d x \\
= & \int_{\Omega}[\nabla u \cdot \nabla a(x)] g(0) u+g(0) \int_{\Omega} a(x)|\nabla u|^{2} d x+\int_{\Omega}[\nabla u \cdot \nabla a] g^{\prime} * u d x \\
& +\int_{\Omega} a(x) \nabla u \cdot g^{\prime} * \nabla u d x .
\end{aligned}
$$

Note that

$$
\begin{aligned}
\int_{\Omega}[\nabla u \cdot \nabla a] g(0) u & +\int_{\Omega}[\nabla u \cdot \nabla a] g^{\prime} * u d x \\
& =g(t) \int_{\Omega}[\nabla u \cdot \nabla a] u+\int_{\Omega} \nabla u \cdot \nabla a \int_{0}^{t} g^{\prime}(t-\tau)\{u(\cdot, \tau)-u(\cdot, t)\} d x .
\end{aligned}
$$


Similarly, we have

$$
\begin{aligned}
& g(0) \int_{\Omega} a(x)|\nabla u|^{2} d x+\int_{\Omega} a(x) \nabla u \cdot \nabla g^{\prime} * u d x \\
& \quad=g(t) \int_{\Omega} a(x)|\nabla u|^{2} d x+\int_{\Omega} a(x) \nabla u \cdot \int_{0}^{t} g^{\prime}(t-\tau)\{\nabla u(\cdot, \tau)-\nabla u(\cdot, t)\} d x .
\end{aligned}
$$

Using the above formulas we conclude that $I_{2}$ may be written as

$$
\begin{aligned}
I_{2}(t)= & g(t) \int_{\Omega}[\nabla u \cdot \nabla a] u+\int_{\Omega}[\nabla u \cdot \nabla a] \int_{0}^{t} g^{\prime}(t-\tau)\{u(\cdot, \tau)-u(\cdot, t)\} d x \\
& +g(t) \int_{\Omega} a(x)|\nabla u|^{2} d x+\int_{\Omega} a(x) \nabla u \cdot \int_{0}^{t} g^{\prime}(t-\tau)\{\nabla u(\cdot, \tau)-\nabla u(\cdot, t)\} d x .
\end{aligned}
$$

From hypotheses $(1.5),(1.7)-(1.8)$ we have that

$$
\begin{aligned}
\int_{\Omega}[\nabla & u \cdot \nabla a] \int_{0}^{t} g^{\prime}(t-\tau)\{u(\cdot, \tau)-u(\cdot, t)\} d \tau d x \\
& \leq c \int_{\Omega} \sqrt{a}|\nabla u|\left\{\int_{0}^{t} g d \tau\right\}^{1 / 2}\left\{\int_{0}^{t} g(t-\tau)|u(\cdot, t)-u(\cdot, \tau)|^{2} d \tau\right\}^{1 / 2} d x \\
& \leq \delta \int_{\Omega} a(x)|\nabla u|^{2} d x+C_{\delta} \int_{0}^{t} \int_{\omega_{\varepsilon}}|u(\cdot, t)-u(\cdot, \tau)|^{2} d x d t .
\end{aligned}
$$

Using similar ideas to estimate the term

$$
\int_{\Omega} a(x) \nabla u \cdot \int_{0}^{t} g^{\prime}(t-\tau)\{\nabla u(\cdot, \tau)-\nabla u(\cdot, t)\} d x d t
$$

we arrive at

$$
\begin{aligned}
I_{2}(t) \leq & C_{\delta} g(t) \int_{\Omega} a(x)|\nabla u|^{2} d x+C_{\delta} g \square \nabla u+\delta \int_{\Omega} a(x)|\nabla u|^{2} d x \\
& +C_{\delta} \int_{0}^{t} \int_{\omega_{\varepsilon}} g^{\prime}(t-\tau)|u(x, t)-u(x, \tau)|^{2} d x d \tau,
\end{aligned}
$$

where $\delta$ is a small parameter to be fixed later and $C_{\delta} \rightarrow \infty$ as $\delta \rightarrow 0$. Finally,

$$
\begin{aligned}
I_{3}(t)= & -\int_{\Omega} a g * \nabla u \cdot \nabla(a g * u)_{t} d x \\
= & -\int_{\Omega} a(x) \int_{0}^{t} g(t-\tau)[\{\nabla u(\cdot, \tau)-\nabla u(\cdot, t)\} \cdot \nabla a](g * u)_{t} \\
& -\int_{0}^{t} g d \tau \int_{\Omega} a(x)[\nabla u \cdot \nabla a](g * u)_{t} d x-\frac{1}{2} \frac{d}{d t} \int_{\Omega} a^{2}|g * \nabla u|^{2} d x \\
= & -\int_{\Omega} a(x) \int_{0}^{t} g(t-\tau)\{\nabla u(\cdot, \tau)-\nabla u(\cdot, t)\} \cdot \nabla a(x)\left\{g(0) u+g^{\prime} * u\right\} d x \\
& -\int_{0}^{t} g d \tau \int_{\Omega} a(x) \nabla u \cdot \nabla a(x)\left\{g(0) u+g^{\prime} * u\right\}-\frac{1}{2} \frac{d}{d t} \int_{\Omega} a^{2}|g * \nabla u|^{2} d x .
\end{aligned}
$$

Using the identity

$$
g(0) u+g^{\prime} * u=g(t)+\int_{0}^{t} g^{\prime}(t-\tau)\{u(\cdot, \tau)-u(\cdot, t)\} d \tau
$$


and since $a(x) \leq 1$, with the same arguments we used to estimate $I_{2}$ we have

$$
\begin{aligned}
-\int_{\Omega} a(x) \int_{0}^{t} g(t-\tau)[\{\nabla u(\cdot, \tau)-\nabla u(\cdot, t)\} \cdot \nabla a] d \tau\left\{g(0) u+g^{\prime} * u\right\} d x \\
\leq C\left\{g \square \nabla u+g(t) \int_{\Omega} a(x)|\nabla u|^{2} d x\right\}+\delta \int_{\Omega} a(x)|\nabla u|^{2} d x+C_{\delta} \int_{\omega_{\varepsilon}}|u|^{2} d x \\
\quad+C_{\delta} \int_{0}^{t} \int_{\omega_{\varepsilon}} g(t-\tau)|u(\cdot, t)-u(\cdot, \tau)|^{2} d x d t
\end{aligned}
$$

and

$$
\begin{array}{rl}
\int_{0}^{t} g & d \tau \int_{\Omega} a(x) \nabla u \cdot \nabla a(x)\left\{g(0) u+g^{\prime} * u\right\} \\
\leq & \delta \int_{\Omega} a(x)|\nabla u|^{2} d x+C_{\delta} \int_{\omega_{\varepsilon}}|u|^{2} d x \\
& +C_{\delta} \int_{0}^{t} \int_{\omega_{\varepsilon}} g(t-\tau)|u(\cdot, t)-u(\cdot, \tau)|^{2} d x d t
\end{array}
$$

from which it follows that

$$
\begin{aligned}
I_{3}(t) \leq & -\frac{1}{2} \frac{d}{d t} \int_{\Omega} a^{2}|g * \nabla u|^{2} d x+C g \square \nabla u+\delta \int_{\Omega} a(x)|\nabla u|^{2} d x \\
& +C_{\delta} \int_{\omega_{\varepsilon}}|u|^{2} d x+C_{\delta} \int_{0}^{t} \int_{\omega_{\varepsilon}} g(t-\tau)|u(\cdot, t)-u(\cdot, \tau)|^{2} d x d t .
\end{aligned}
$$

Since

$$
-I_{1}=I_{2}+I_{3}
$$

our conclusion follows by substitution of the relations for $I_{i}, i=1,2,3$, into the above identity. The proof is not complete

Lemma 4.2. With the same hypotheses as Lemma 4.1 we have that the solution of Eqs. (1.1)-(1.3) satisfies

$$
\begin{aligned}
\frac{d}{d t} \int_{\Omega} a_{\Omega} d x \leq & \int_{\Omega} a(x)\left|u_{t}\right|^{2} d x-c_{0} \int_{\Omega} a(x)|\nabla u|^{2} d x+c \int_{\omega_{\varepsilon}}|u|^{2} d x \\
& +C_{\delta} \int_{0}^{t} \int_{\omega_{\varepsilon}} g(t-\tau)|u(\cdot, t)-u(\cdot, \tau)|^{2} d x d t
\end{aligned}
$$

Proof. Let us multiply Eq. (1.1) by $a(x) u(x, t)$ to get

$$
\underbrace{\int_{\Omega} a u_{t t} u d x}_{:=I_{1}} \underbrace{-\int_{\Omega} a(x) u \Delta u d x}_{:=I_{2}}+\underbrace{\int_{\Omega} \operatorname{div}\{a g * \nabla u\} a u d x}_{:=I_{3}}=0 .
$$

Note that

$$
I_{1}(t)=\frac{d}{d t} \int_{\Omega} a u_{t} u d x-\int_{\Omega} a(x)\left|u_{t}\right|^{2} d x
$$


On the other hand,

$$
\begin{aligned}
I_{2}(t) & =\int_{\Omega} \nabla u \nabla\{a u\} d x \\
& =\int_{\Omega} u \nabla u \nabla a d x+\int_{\Omega} a(x)|\nabla u|^{2} d x \\
& =-\frac{1}{2} \int_{\Omega} \Delta a(x)|u|^{2} d x+\int_{\Omega} a(x)|\nabla u|^{2} d x .
\end{aligned}
$$

Finally,

$$
\begin{aligned}
I_{3}(t)= & -\int_{\Omega} a(x) g * \nabla u \cdot \nabla\{a u\} d x \\
= & -\int_{\Omega} a(x)[g * \nabla u \cdot \nabla a] u d x-\int_{\Omega} a^{2} g * \nabla u \cdot \nabla u d x \\
= & -\int_{\Omega} a(x)[g * \nabla u \cdot \nabla a] u d x+\int_{0}^{t} g d \tau \int_{\Omega} a^{2}|\nabla u|^{2} d x \\
& -\int_{\Omega} a^{2} \int_{0}^{t} g(t-\tau)\{\nabla u(\cdot, \tau)-u(\cdot, t)\} \cdot \nabla u d x .
\end{aligned}
$$

Summing up $I_{1}, I_{2}$, and $I_{3}$ we get

$$
\begin{aligned}
\frac{d}{d t} \int_{\Omega} a u u_{t} d x= & \int_{\Omega} a(x)\left|u_{t}\right|^{2} d x-\int_{\Omega} a\left(1-a(x) \int_{0}^{t} g d \tau\right)|\nabla u|^{2} d x+\int_{\Omega}(\Delta a)|u|^{2} d x \\
& +\int_{\Omega} a(x) \int_{0}^{t} g(t-\tau)\{\nabla u(\cdot, \tau)-\nabla u(\cdot, t)\} \nabla a(x) u d \tau d x \\
& +\int_{\Omega} a^{2} \int_{0}^{t} g(t-\tau)\{\nabla u(\cdot, \tau)-\nabla u(\cdot, t)\} d \tau \nabla u d x
\end{aligned}
$$

Using $|\Delta a(x)| \leq C$ our conclusion follows.

The following lemma is proved by Lions [13]. For convenience we rewrite it here.

Lemma 4.3. Let us denote by $q_{k}$ a $C^{1}$-function. Then any strong solution $\left(u \in C^{i}(0, T\right.$; $\left.H^{2-i}(\Omega)\right)$ for $\left.i=0,1,2\right)$ of the wave equation

$$
\begin{gathered}
u_{t t}-\Delta u=f, \\
u(x, t)=0 \quad \text { on } \Gamma \times] 0 \infty[,
\end{gathered}
$$

satisfies the following identity:

$$
\begin{gathered}
\frac{d}{d t} \int_{\Omega} u_{t} q_{k} \frac{\partial u}{\partial x_{k}} d x=\int_{\Omega} f q_{k} \frac{\partial u}{\partial x_{k}} d x+\frac{1}{2} \int_{\Gamma} q_{k} \nu_{k}\left|\frac{\partial u}{\partial \nu}\right|^{2} d \Gamma+\int_{\Gamma} \frac{\partial u}{\partial \nu} q_{k} \frac{\partial u}{\partial x_{k}} d \Gamma \\
+\frac{1}{2} \int_{\Omega} \frac{\partial q_{k}}{\partial x_{k}}\left\{\left|u_{t}\right|^{2}+|\nabla u|^{2}\right\} d x+\int_{\Omega} \nabla u \cdot \nabla q_{k} \frac{\partial u}{\partial x_{k}} d x
\end{gathered}
$$

Proof. Let us multiply Eq. (4.1) by $q_{k} \frac{\partial u}{\partial x_{k}}$ to get

$$
\int_{\Omega}\left\{u_{t t}-\Delta u\right\} q_{k} \frac{\partial u}{\partial x_{k}} d x=\int_{\Omega} f q_{k} \frac{\partial u}{\partial x_{k}} d x .
$$


It is easy to see that

$$
\begin{aligned}
\int_{\Omega} u_{t t} q_{k} \frac{\partial u}{\partial x_{k}} d x & =\frac{d}{d t} \int_{\Omega} u_{t} q_{k} \frac{\partial u}{\partial x_{k}} d x-\int_{\Omega} u_{t} q_{k} \frac{\partial u_{t}}{\partial x_{k}} d x \\
& =\frac{d}{d t} \int_{\Omega} u_{t} q_{k} \frac{\partial u}{\partial x_{k}} d x-\frac{1}{2} \int_{\Omega} q_{k} \frac{\partial\left|u_{t}\right|^{2}}{\partial x_{k}} d x \\
& =\frac{d}{d t} \int_{\Omega} u_{t} q_{k} \frac{\partial u}{\partial x_{k}} d x+\frac{1}{2} \int_{\Omega} \frac{\partial q_{k}}{\partial x_{k}}\left|u_{t}\right|^{2} d x
\end{aligned}
$$

On the other hand,

$$
\begin{aligned}
-\int_{\Omega} \Delta u q_{k} \frac{\partial u}{\partial x_{k}} d x= & -\int_{\Gamma} \frac{\partial u}{\partial \nu} q_{k} \frac{\partial u}{\partial x_{k}} d \Gamma+\int_{\Omega} \nabla u \cdot \nabla q \frac{\partial u}{\partial x_{k}} d x+\int_{\Omega} \nabla u \cdot q \frac{\partial \nabla u}{\partial x_{k}} d x \\
= & -\int_{\Gamma} \frac{\partial u}{\partial \nu} q_{k} \frac{\partial u}{\partial x_{k}} d \Gamma+\int_{\Omega} \nabla u \cdot \nabla q \frac{\partial u}{\partial x_{k}} d x+\frac{1}{2} \int_{\Omega} q_{k} \frac{\partial|\nabla u|^{2}}{\partial x_{k}} d x \\
= & -\int_{\Gamma} \frac{\partial u}{\partial \nu} q_{k} \frac{\partial u}{\partial x_{k}} d \Gamma+\int_{\Omega} \nabla u \cdot \nabla q \frac{\partial u}{\partial x_{k}} d x+\frac{1}{2} \int_{\Gamma} q_{k} \nu_{k}|\nabla u|^{2} d \Gamma \\
& -\frac{1}{2} \int_{\Omega} \frac{\partial q_{k}}{\partial x_{k}}|\nabla u|^{2} d x
\end{aligned}
$$

Since $u(x, t)=0$ on $\Gamma$, we have that

$$
\frac{\partial u}{\partial x_{k}}=\nu_{k} \frac{\partial u}{\partial \nu}
$$

from which our conclusion follows.

LEMMA 4.4. Let us take $q_{k}=a^{2}(x) h_{k}$, where $h_{k} \in C^{2}(\bar{\Omega})$ is such that $h_{k}=\nu_{k}$ on $\Gamma$. Then we have that

$$
\begin{aligned}
-\frac{d}{d t} \int_{\Omega} a^{2}(x) u_{t} h_{k} \frac{\partial u}{\partial x_{k}} d x \leq & -\frac{1}{2} \int_{\Gamma_{0}}\left|\frac{\partial u}{\partial \nu}\right|^{2} d \Gamma+C \int_{\Omega} a(x)\left\{\left|u_{t}\right|^{2}+|\nabla u|^{2}\right\} d x \\
& +C \int_{\Omega} a(x)|h(x) \operatorname{div}\{a g * \nabla u\}|^{2} d x,
\end{aligned}
$$

for any solution of Eqs. (1.1)-(1.3).

Proof. From Lemma 4.3 applied to $q_{k}=a^{2}(x) h_{k}$ we have

$$
\begin{aligned}
& -\frac{d}{d t} \int_{\Omega} a^{2} h_{k} u_{t} \frac{\partial u}{\partial x_{k}} d x=-\int_{\Omega}-f a^{2}(x) h_{k} \frac{\partial u}{\partial x_{k}} d x=\int_{\Gamma} a^{2}(x) h_{k} \nu_{k}\left|\frac{\partial u}{\partial \nu}\right|^{2} d x \\
& \quad+\frac{1}{2} \int_{\Omega} \frac{\partial\left\{a^{2}(x) h_{k}\right\}}{\partial x_{k}}\left\{\left|u_{t}\right|^{2}-|\nabla u|^{2}\right\} d x+\int_{\Omega} \nabla u \cdot \nabla a^{2} h_{k} \frac{\partial u}{\partial x_{k}} d x .
\end{aligned}
$$

Since $a=1$ on $\Gamma_{0}$ and $h_{k}=\nu_{k}$ on $\Gamma$, using the Cauchy-Schwarz inequality, our conclusion follows.

Let us denote by $\mathcal{L}_{N}(t)$ the functional

$$
\mathcal{L}_{N}(t)=N E(t)+I(t)+\frac{g(0)}{2} \int_{\Omega} a(x) u u_{t} d x-\delta_{0} \int_{\Omega} a^{2}(x) u_{t} h_{k} \frac{\partial u}{\partial x_{k}} d x .
$$

Under these conditions we get 
LEMMA 4.5. Under the above notation we have

$$
\begin{aligned}
\frac{d}{d t} \mathcal{L}_{N}(t) \leq & -\kappa_{0} \int_{\Omega} a(x)\left\{\left|u_{t}\right|^{2}+|\nabla u|^{2}\right\} d x-\frac{N}{2}\left\{g \square \nabla u+g(t) \int_{\Omega} a(x)|\nabla u|^{2} d x\right\} \\
& -\frac{\delta_{0}}{2} \int_{\Gamma}\left|\frac{\partial u}{\partial \nu}\right|^{2} d \Gamma+\left.C \delta_{0} \int_{\Omega} a(x)\left|h(x) \operatorname{div}\{a g * \nabla u\}^{2} d x+C \int_{\omega_{\varepsilon}}\right| u\right|^{2} d x \\
& +C \int_{0}^{t} \int_{\omega_{\varepsilon}} g(t-\tau)|u(x, t)-u(x, \tau)|^{2} d x d \tau .
\end{aligned}
$$

Proof. From Lemma 4.1 and Lemma 4.2 we get that

$$
\begin{aligned}
\frac{d}{d t}\left\{I(t)+\frac{g(0)}{2} \int_{\Omega} a(x) u u_{t} d x\right\} \leq & -\frac{g(0)}{2} \int_{\Omega} a(x)\left|u_{t}\right|^{2} d x-(C-\delta) \int_{\Omega} a(x)|\nabla u|^{2} d x \\
& +C \int_{\omega_{\varepsilon}}|u|^{2} d x+C_{\delta}\left\{g \square \nabla u+g(t) \int_{\Omega} a(x)|\nabla u|^{2} d x\right\} \\
& +C \int_{0}^{t} \int_{\omega_{\varepsilon}} g(t-\tau)|u(x, t)-u(x, \tau)|^{2} d x d \tau .
\end{aligned}
$$

So, taking $\delta$ small enough, we get that there exists a positive constant $k_{0}$ such that

$$
\begin{aligned}
\frac{d}{d t}\left\{I(t)+\frac{g(0)}{2} \int_{\Omega} a(x) u u_{t} d x\right\} \leq & -2 \kappa_{0}\left\{\int_{\Omega} a(x)\left|u_{t}\right|^{2} d x+\int_{\Omega} a(x)|\nabla u|^{2} d x\right\} \\
& +C \int_{\omega_{\varepsilon}}|u|^{2} d x+C_{\delta}\left\{g \square \nabla u+g(t) \int_{\Omega} a(x)|\nabla u|^{2} d x\right\} \\
& +C \int_{\Omega}^{t} \int_{\omega_{\varepsilon}} g(t-\tau)|u(x, t)-u(x, \tau)|^{2} d x d \tau .
\end{aligned}
$$

Using Lemma 2.2, Lemma 4.4, and the above inequality we arrive at

$$
\begin{aligned}
\frac{d}{d t} \mathcal{L}_{N}(t) \leq & -\kappa_{0}\left\{\int_{\Omega} a(x)\left|u_{t}\right|^{2} d x+\int_{\Omega} a(x)|\nabla u|^{2} d x\right\}-\frac{\varepsilon}{2} \int_{\Gamma}\left|\frac{\partial u}{\partial \nu}\right|^{2} d \Gamma \\
& -\left(N-C_{\delta}\right)\left\{g \square \nabla u+g(t) \int_{\Omega} a(x)|\nabla u|^{2} d x\right\}+C \int_{\omega_{\varepsilon}}|u|^{2} d x \\
& +C \varepsilon \int_{\Omega} a(x)|h(x) \operatorname{div}\{a g * \nabla u\}|^{2} d x \\
& +C \int_{0}^{t} \int_{\omega_{\varepsilon}} g(t-\tau)|u(x, t)-u(x, \tau)|^{2} d x d \tau .
\end{aligned}
$$

Therefore, taking $N>2 C_{\delta}$, we get

$$
\begin{aligned}
\frac{d}{d t} \mathcal{L}_{N}(t) \leq & -\kappa_{0}\left\{\int_{\Omega} a(x)\left|u_{t}\right|^{2} d x+\int_{\Omega} a(x)|\nabla u|^{2} d x\right\}-\frac{\varepsilon}{2} \int_{\Gamma}\left|\frac{\partial u}{\partial \nu}\right|^{2} d \Gamma \\
& -\frac{N}{2}\left\{g \square \nabla u+g(t) \int_{\Omega} a(x)|\nabla u|^{2} d x\right\}+C \int_{\omega_{\varepsilon}}|u|^{2} d x \\
& +C \delta_{0} \int_{\Omega} a(x)|h(x) \operatorname{div}\{a g * \nabla u\}|^{2} d x \\
& +\int_{0}^{t} \int_{\omega_{\varepsilon}} g(t-\tau) \mid u(x, t)=u(x, \tau)^{2} d x d \tau .
\end{aligned}
$$


This proof is now complete.

LEMmA 4.6. Let us suppose that $u$ is the weak solution of $(1.1)-(1.3)$. Then there exists a positive constant $C$, independent of $T$, such that

$$
\begin{aligned}
\int_{0}^{T} \int_{\Omega} a(x)|\operatorname{div}\{a g * \nabla u\}|^{2} d x d t \leq & C \int_{0}^{T} \int_{\Omega} a(x)\left\{\left|u_{t}\right|^{2}+|\nabla u|^{2}\right\} d x d t \\
& +C \int_{\Omega}^{T} g(t) d t E(0), \\
\int_{0}^{T} \int_{\Omega}|\operatorname{div}\{a g * \nabla u\}|^{2} d x d t \leq & C \int_{0}^{T} E(t) d t+C \int_{0}^{T} g(t) d t E(0) .
\end{aligned}
$$

Proof. Note that

$$
\operatorname{div}\{a g * \nabla u\}=\nabla a \cdot \nabla u+a g * \Delta u
$$

As in the proof of Lemma 3.2, $v=\sqrt{a} g * \Delta u$ satisfies

$$
-v+a(x) g * v=\sqrt{a} G,
$$

where $G$ is given by (3.3). Using similar arguments, we conclude that

$$
\begin{aligned}
\|v\|_{L^{2}\left(0, T ; L^{2}\right)}^{2} & \leq \int_{0}^{T} \int_{\Omega} a(x)|G|^{2} d x d t \\
& \leq \int_{0}^{T} \int_{\Omega} a(x)\left\{\left|u_{t}\right|^{2}+|\nabla u|^{2}\right\} d x d t+C \int_{0}^{T} g d t E(0) .
\end{aligned}
$$

Therefore, it follows that

$$
\begin{aligned}
& \int_{0}^{T} \int_{\Omega} a(x)|\operatorname{div}\{a g * \nabla u\}|^{2} d x d t \\
& \quad \leq C \int_{0}^{T} \int_{\Omega} a(x)\left\{\left|u_{t}\right|^{2}+|\nabla u|^{2}\right\} d x d t+C \int_{0}^{T} g(t) d t E(0),
\end{aligned}
$$

for a positive constant $C$. The proof is now complete.

LEMMA 4.7. Let us suppose that $\varphi$ is a weak solution of the wave equation

$$
\begin{gathered}
\varphi_{t t}-\Delta \varphi=0 \\
\varphi(x, 0)=\varphi_{0}, \quad \varphi_{t}=\varphi_{1}, \\
\varphi(x, t)=0 \quad \text { on } \Sigma=\Gamma \times] 0, \infty[.
\end{gathered}
$$

for any $\left(\varphi_{0}, \varphi_{1}\right) \in H_{0}^{1}(\Omega) \times L^{2}(\Omega)$ where

$$
R\left(x_{0}\right)=\max _{x \in \bar{\Omega}}\left|\sum_{k=1}^{n}\left(x_{k}-x_{k}^{0}\right)^{2}\right|^{1 / 2}
$$

Proof. See [13], Lemma 2.3, Chapter VIII, p. 411.

Our next step is to estimate the term $\int_{\omega}|u|^{2} d x$. To do this we will use the following lemma. 
LEMmA 4.8. Let us suppose that $u$ is a weak solution of (1.1)-(1.3). Then for any $\varepsilon>0$ there exists a positive constant $C_{\varepsilon}$ for which we have

$$
\begin{aligned}
\int_{0}^{T} \int_{\Omega}|u|^{2} d x d t \leq & C_{\varepsilon}\left\{\int_{0}^{T} g(t) \int_{\Omega} a(x)|\nabla u|^{2} d x d t+\int_{0}^{T} g \square \nabla u d t\right\} \\
& +\varepsilon \int_{0}^{T} \int_{\Omega} a(x)\left\{|\nabla u|^{2}+\left|u_{t}\right|^{2}+|\operatorname{div} a g * \nabla u|^{2}\right\} d x d t \\
\int_{0}^{T} \int_{\Omega}|g * \nabla u|^{2} d x d t \leq & C_{\varepsilon}\left\{\int_{0}^{T} g(t) \int_{\Omega} a(x)|\nabla u|^{2} d x d t+\int_{0}^{T} g \square \nabla u d t\right\} \\
+ & \varepsilon \int_{0}^{T} \int_{\Omega} a(x)\left\{|\nabla u|^{2}+\left|u_{t}\right|^{2}+\left.\operatorname{div} a g * \nabla u\right|^{2}\right\} d x d t
\end{aligned}
$$

and

$$
\begin{aligned}
& \int_{0}^{T} \int_{0}^{t} \int_{\Omega} g(\sigma-t)|u(x, \sigma)-u(x, t)|^{2} d x d \sigma d t \\
& \leq C_{\varepsilon}\left\{\int_{0}^{T} g(t) \int_{\Omega} a(x)|\nabla u|^{2} d x d t+\int_{0}^{T} g \square \nabla u d t\right\} \\
& \quad+\varepsilon \int_{0}^{T} \int_{\Omega} a(x)\left\{|\nabla u|^{2}+\left|u_{t}\right|^{2}+|\operatorname{div} a g * \nabla u|^{2}\right\} d x d t
\end{aligned}
$$

provided $T$ is large enough.

Proof. We argue by contradiction. Suppose that there exists $\varepsilon_{0}>0$ and a sequence of functions such that

$$
\begin{aligned}
\int_{0}^{T} \int_{\Omega}\left|u^{\nu}\right|^{2} d x d t \geq & \nu\left\{\int_{0}^{T} g(t) \int_{\Omega} a(x)\left|\nabla u^{\nu}\right|^{2} d x d t+\int_{0}^{T} g \square \nabla u^{\nu} d t\right\} \\
& +\varepsilon_{0} \int_{0}^{T} \int_{\Omega} a(x)\left\{\left|\nabla u^{\nu}\right|^{2}+\left|u_{t}^{\nu}\right|^{2}+|\operatorname{div} a g * \nabla u|^{2}\right\} d x d t
\end{aligned}
$$

for $\nu \rightarrow \infty$. By the linearity of the problem we may suppose that

$$
\int_{0}^{T} \int_{\Omega}\left|u^{\nu}\right|^{2} d x d t=1 \quad \forall \nu \in \mathbb{N}
$$

So, we get that

$$
g(t) a(x)\left|\nabla u^{\nu}\right|^{2}+\int_{0}^{t} a(\cdot) g(t-\tau)\left|u^{\nu}(\cdot, \tau)-u^{\nu}(\cdot, t)\right|^{2} d \tau \rightarrow 0 \quad \text { strongly in } L^{1}(] 0, \infty[\times \Omega) .
$$

Let us decompose $u^{\nu}$ into

$$
u^{\nu}=w^{\nu}+v^{\nu}
$$

where

$$
\begin{gathered}
\left.w_{t t}^{\nu}-\Delta w^{\nu}=-\operatorname{div}\left\{a g * \nabla u^{\nu}\right\} \quad \text { (bounded in } L^{2}\left(0, T ; L^{2}(\Omega)\right)\right), \\
w^{\nu}(x, 0)=0, \quad w_{t}^{\nu}(x, 0)=0 \quad \text { in } \Omega \\
\left.w^{\nu}(x, t)=0 \quad \text { on } \Gamma \times\right] 0, \infty[
\end{gathered}
$$


and

$$
\begin{gathered}
v_{t t}^{\nu}-\Delta v^{\nu}=0, \\
v^{\nu}(x, 0)=u^{\nu}(x, 0), \quad v_{t}^{\nu}(x, 0)=u_{t}^{\nu}(x, 0) \quad \text { in } \Omega, \\
\left.v^{\nu}(x, t)=0 \quad \text { on } \Gamma \times\right] 0, \infty[.
\end{gathered}
$$

From (4.6) and (4.7) it follows that $u^{\nu}$ is bounded in

$$
W^{1, \infty}\left(0, T ; L^{2}(\omega)\right) \cap L^{\infty}\left(0, T ; H^{1}(\omega)\right) .
$$

Note that $w^{\nu}$ is also bounded in

$$
W^{1, \infty}\left(0, T ; L^{2}(\Omega)\right) \cap L^{\infty}\left(0, T ; H_{0}^{1}(\Omega)\right) .
$$

Thereby, we conclude that $v^{\nu}=u^{\nu}-w^{\nu}$ satisfies

$$
\begin{aligned}
& v_{t}^{\nu} \text { is bounded in } L^{2}\left(0, T ; L^{2}(\omega)\right), \\
& v^{\nu} \text { is bounded in } L^{2}\left(0, T ; H^{1}(\omega)\right) .
\end{aligned}
$$

Using Lemma 4.7 we have

$$
\left(u^{\nu}(\cdot, 0), u_{t}^{\nu}(\cdot, 0)\right)=\left(v^{\nu}(\cdot, 0), v_{t}^{\nu}(\cdot, 0)\right) \text { is bounded in } H_{0}^{1}(\Omega) \times L^{2}(\Omega),
$$

which implies that

$$
v^{\nu} \text { is bounded in } W^{1, \infty}\left(0, T ; L^{2}(\Omega)\right) \cap L^{\infty}\left(0, T ; H_{0}^{1}(\Omega)\right) .
$$

Hence

$$
u^{\nu}=w^{\nu}+v^{\nu} \text { is bounded in } W^{1, \infty}\left(0, T ; L^{2}(\Omega)\right) \cap L^{\infty}\left(0, T ; H_{0}^{1}(\Omega)\right) .
$$

Therefore, there exists a subsequence (which we still denote in the same way) and a function $u \in W^{1, \infty}\left(0, T ; L^{2}(\Omega)\right)$ such that

$$
u^{\nu} \rightarrow u \quad \text { weak } * \text { in } W^{1, \infty}\left(0, T ; L^{2}(\Omega)\right)
$$

and satisfying

$$
\begin{gathered}
u_{t t}-\Delta u=0, \\
u(x, 0)=u_{0}(x), \quad u_{t}(x, 0)=u_{1}(x) \quad \text { in } \Omega, \\
u(x, t)=0 \quad \text { on } \Gamma \times] 0, T[.
\end{gathered}
$$

From (4.8) we conclude that

$$
\left.u=0 \quad \text { on } \omega_{\varepsilon} \times\right] 0, T[.
$$

Using Holmgren's Theorem for $T>2 \operatorname{diam}\left(\Omega \backslash \omega_{\varepsilon}\right)$, we get that $u=0$ on $\left.\Omega \times\right] 0, T[$. But this is contradictory with (4.7) since, due to the compactness of the embedding $H^{1}\left(\Omega \times 0, T[) \subset L^{2}(\Omega \times] 0, T[)\right.$, the sequence $u^{\nu}$ converges strongly in $L^{2}(\Omega \times] 0, T[)$. This contradiction proves the first inequality. To prove the other we use similar arguments. Thereby, our conclusion follows. 
Using the inequalities (4.3), (4.5), Lemma 4.8 and taking $\varepsilon>0$ small enough we arrive at

$$
\mathcal{L}_{N}(T)-\mathcal{L}_{N}(0) \leq-\kappa_{0} \int_{0}^{T} \mathcal{M}(t) d t+C \varepsilon E(0)+C \varepsilon \int_{0}^{T} E(t) d t
$$

for $N>2 C$, where by $\mathcal{M}$ we are denoting

$$
\mathcal{M}(t)=\int_{\Omega} a(x)\left\{\left|u_{t}\right|^{2}+|\nabla u|^{2}\right\} d x+g \square \nabla u+\int_{\Gamma_{0}}\left|\frac{\partial u}{\partial \nu}\right|^{2} d \Gamma .
$$

Now we have the conditions to prove the main result of this paper.

Proof of Theorem 1.1. We will suppose that the initial data belongs to $H^{2}(\Omega) \cap H_{0}^{1}(\Omega)$ $\times H_{0}^{1}(\Omega)$. Our conclusion will follow using standard density arguments. Using Lemma 4.3 for $q=x-x^{0}$ we conclude that

$$
\begin{aligned}
-\frac{d}{d t} \int_{\Omega} u_{t} q_{k} \frac{\partial u}{\partial x_{k}} d x= & -\int_{\Omega} f q_{k} \frac{\partial u}{\partial x_{k}} d x+\frac{1}{2} \int_{\Omega} \frac{\partial q_{k}}{\partial x_{k}}\left\{\left|u_{t}\right|^{2}-|\nabla u|^{2}\right\} d x \\
& +\int_{\Omega} \nabla u \cdot \nabla q_{k} \frac{\partial u}{\partial x_{k}} d x-\frac{1}{2} \int_{\Gamma} q_{k} \nu_{k}\left|\frac{\partial u}{\partial \nu}\right|^{2}
\end{aligned}
$$

from which it follows that

$$
\begin{aligned}
-\frac{d}{d t} \int_{\Omega} u_{t} q_{k} \frac{\partial u}{\partial x_{k}} d x= & -\int_{\Omega} f q_{k} \frac{\partial u}{\partial x_{k}} d x+\frac{n}{2} \int_{\Omega}\left\{\left|u_{t}\right|^{2}-|\nabla u|^{2}\right\} d x \\
& +\int_{\Omega}|\nabla u|^{2} d x-\frac{1}{2} \int_{\Gamma} q_{k} \nu_{k}\left|\frac{\partial u}{\partial \nu}\right|^{2}
\end{aligned}
$$

which implies that

$$
\begin{aligned}
-\frac{d}{d t} \int_{\Omega} u_{t} q_{k} \frac{\partial u}{\partial x_{k}} d x= & -\int_{\Omega} f q_{k} \frac{\partial u}{\partial x_{k}} d x+\frac{n-1}{2} \int_{\Omega}\left\{\left|u_{t}\right|^{2}-|\nabla u|^{2}\right\} d x \\
& +\frac{1}{2} \int_{\Omega}\left\{\left|u_{t}\right|^{2}+|\nabla u|^{2}\right\} d x-\frac{1}{2} \int_{\Gamma} q k \nu_{k}\left|\frac{\partial u}{\partial \nu}\right|^{2}
\end{aligned}
$$

Multiplying Eq. (1.1) by $u$ we get

$$
\frac{d}{d t} \int_{\Omega} u u_{t} d x=\int_{\Omega}\left\{\left|u_{t}\right|^{2}-|\nabla u|^{2}\right\} d x+\int_{\Omega} a g * \nabla u \cdot \nabla u d x .
$$

Inserting this identity into (4.10) we have

$$
\begin{aligned}
-\frac{d}{d t} \int_{\Omega} u_{t} q_{k} \frac{\partial u}{\partial x_{k}} d x= & -\int_{\Omega} f q_{k} \frac{\partial u}{\partial x_{k}} d x+\frac{n-1}{2} \frac{d}{d t} \int_{\Omega} u u_{t} d x \\
& -\frac{n-1}{2} \int_{\Omega} a g * \nabla u \cdot \nabla u d x+\frac{1}{2} \int_{\Omega}\left\{\left|u_{t}\right|^{2}+|\nabla u|^{2}\right\} d x \\
& -\frac{1}{2} \int_{\Gamma} q_{k} \nu_{k}\left|\frac{\partial u}{\partial \nu}\right|^{2}
\end{aligned}
$$


from which we have

$$
\begin{aligned}
& \frac{d}{d t} \underbrace{\left\{-\int_{\Omega} u_{t} q_{k} \frac{\partial u}{\partial x_{k}} d x-\frac{n-1}{2} \int_{\Omega} u u_{t} d x\right\}}_{:=X(t)} \\
& =-\int_{\Omega} f q_{k} \frac{\partial u}{\partial x_{k}} d x-\frac{n-1}{2} \int_{\Omega} a g * \nabla u \cdot \nabla u d x \\
& +\frac{1}{2} \int_{\Omega}\left\{\left|u_{t}\right|^{2}+|\nabla u|^{2}\right\} d x-\frac{1}{2} \int_{\Gamma} q_{k} \nu_{k}\left|\frac{\partial u}{\partial \nu}\right|^{2} .
\end{aligned}
$$

Integrating over $[0, T]$ we get

$$
\begin{aligned}
X(T)-X(0)= & -\int_{0}^{T} \int_{\Omega} f q_{k} \frac{\partial u}{\partial x_{k}} d x d t-\frac{n-1}{2} \int_{0}^{T} \int_{\Omega} a g * \nabla u \cdot \nabla u d x d t \\
& +\frac{1}{2} \int_{0}^{T} \int_{\Omega}\left\{\left|u_{t}\right|^{2}+|\nabla u|^{2}\right\} d x d t-\frac{1}{2} \int_{0}^{T} \int_{\Gamma} q k \nu_{k}\left|\frac{\partial u}{\partial \nu}\right|^{2} d t
\end{aligned}
$$

Since

$$
X(T) \leq C E(T), \quad X(0) \leq C E(0),
$$

and using

$$
\int_{0}^{T} E(t) d t \leq C\left\{\int_{0}^{T} \int_{\Omega}\left\{\left|u_{t}\right|^{2}+|\nabla u|^{2}\right\} d x d t+\int_{0}^{T} g \square \nabla u d t\right\},
$$

together with inequality (4.11) we conclude that

$$
\int_{0}^{T} E(t) d t \leq C \int_{0}^{T} \mathcal{M}(t) d t+C\{E(T)+E(0)\} .
$$

From the energy identity we get

$$
E(0) \leq E(T)+\int_{0}^{T} \mathcal{M}(t) d t
$$

Therefore, there exists a positive constant $C_{1}$ such that

$$
\int_{0}^{T} E(t) d t \leq C_{1} \int_{0}^{T} \mathcal{M}(t) d t+C_{1} E(T) .
$$

Since $E(t)$ is a decreasing function we have that

$$
E(T) \leq \frac{1}{T} \int_{0}^{T} E(t) d t
$$

Inserting the above inequality into (4.13) we get

$$
\left(1-\frac{C}{T}\right) \int_{0}^{T} E(t) d t \leq C_{1} \int_{0}^{T} \mathcal{M}(t) d t .
$$

On the other hand, it is not difficult to see that

$$
c_{0} E(t) \leq \mathcal{L}(t) \leq c_{1} E(t) .
$$


Therefore, using (4.9), (4.14), and (4.15) we conclude that

$$
\begin{aligned}
\mathcal{L}(T)-\mathcal{L}(0) & \leq-\kappa_{0} \int_{0}^{T} \mathcal{M}(t) d t+C \varepsilon E(0)+C \varepsilon \int_{0}^{T} E(t) d t \quad(\text { using }(4.12)) \\
& \leq-\kappa_{0} \int_{0}^{T} \mathcal{M}(t) d t+C \varepsilon\left\{E(T)+\int_{0}^{T} \mathcal{M}(t) d t\right\}+C \varepsilon \int_{0}^{T} E(t) d t \\
& \leq-\kappa_{1} \int_{0}^{T} \mathcal{L}(t) d t,
\end{aligned}
$$

provided $\varepsilon$ is small enough. Using inequality (4.15) we can establish that

$$
\int_{0}^{T} \mathcal{L}(t) d t \geq c \int_{0}^{T} E(t) d t \geq c T E(T) \geq c_{2} T \mathcal{L}(T),
$$

which implies that

$$
\mathcal{L}(T)-\mathcal{L}(0) \leq-\kappa_{1} c T \mathcal{L}(T)
$$

This is equivalent to

$$
\mathcal{L}(T) \leq \frac{1}{1+C T} \mathcal{L}(0)
$$

Repeating the above process from $T$ to $2 T$ we get that

$$
\mathcal{L}(2 T) \leq \frac{1}{1+C T} \mathcal{L}(T) \leq \frac{1}{(1+C T)^{2}} \mathcal{L}(0)
$$

In general we have that

$$
\mathcal{L}(n T) \leq \frac{1}{(1+C T)^{n}} \mathcal{L}(0)
$$

Since any number $t$ can be written as $t=n T+r$ where $r<T$ and $E(t)$ is a decreasing function, from (4.15) we arrive at

$$
\mathcal{L}(t) \leq c \mathcal{L}(t-r) \leq \frac{C}{(1+C T)^{(t-r) / T}} \mathcal{L}(0) \leq c_{0} e^{-\gamma t} \mathcal{L}(0),
$$

where $\gamma=\frac{\ln (1+C T)}{T}$, from which the exponential decay follows.

\section{References}

[1] C. Bardos, G. Lebeau and J. Rauch, Sharp sufficient conditions for the observation, control and stabilization of waves from the boundary, SIAM Journal of Control and Optimization 30, 1024-1065 (1992)

[2] C. M. Dafermos, An abstract Volterra equation with application to linear viscoelasticity, J. Differential Equations 7, 554-589 (1970)

[3] G. Dassios and F. Zafiropoulos, Equipartition of energy in linearized 3-d viscoelasticity, Quart. Appl. Math. 48, 715-730 (1990)

[4] J. M. Greenberg and $\mathrm{Li}$ Tatsien, The effect of the boundary damping for the quasilinear wave equation, J. Differential Equations 52, 66-75 (1984)

[5] M. A. Horn and I. Lasiecka, Uniform decay of weak solutions to a von Kármán plate with nonlinear boundary dissipation, Differential Integral Equations 7, 885-908 (1994)

[6] F. A. Khodja, A. Benabdallah, and D. Teniou, Stabilisation frontière et interne du système de la Thermoélasticité, Reprint de l'équipe de Mathématiques de Besançon 95/32

[7] V. Komornik and E. Zuazua, A direct method for the boundary stabilization of the wave equation, J. Math. Pures et Appl. 69, 33-54 (1990) 
[8] V. Komornik, Rapid boundary stabilization of the wave equation, SIAM J. Control and Optimization 29, 197-208 (1991)

[9] J. E. Lagnese, Asymptotic energy estimates for Kirchhoff plates subject to weak viscoelastic damping, International Series of Numerical Mathematics, Vol. 91, Birkhäuser-Verlag, Basel, 1989

[10] I. Lasiecka and R. Triggiani, Exact controllability and uniform stabilization of Euler-Bernoulli equations with boundary control only in $\left.\Delta w\right|_{\Sigma}$, Bollettino Un. Mat. Ital. $\mathrm{B}(7) \mathbf{5}, 665-702$ (1991)

[11] I. Lasiecka, Global uniform decay rates for the solution to the wave equation with nonlinear boundary conditions, Applicable Analysis 47, 191-212 (1992)

[12] I. Lasiecka, Exponential decay rates for the solutions of Euler-Bernoulli equations with boundary dissipation occurring in the moments only, J. Differential Equations 95, 169-182 (1992)

[13] J. L. Lions, Contrôlabilité exacte, perturbations et stabilisation de systèmes distribués, Tome 1, Masson, Paris, 1988

[14] K. Liu and Z. Liu, Exponential decay of energy of the Euler-Bernoulli beam with locally distributed Kelvin-Voigt damping, SIAM Control and Optimization 36, 1086-1098 (1998)

[15] J. Muñoz Rivera, Asymptotic behaviour in linear viscoelasticity, Quart. Appl. Math. 52, 629-648 (1994)

[16] J. Muñoz Rivera, Global smooth solution for the Cauchy problem in nonlinear viscoelasticity, Differential Integral Equations 7, 257-273 (1994)

[17] J. E. Muñoz Rivera and M. de Lacerda Oliveira, Stability in inhomogeneous and anisotropic thermoelasticity, Bollettino Un. Mat. Ital. A(7) 11, 115-127 (1997)

[18] M. Nakao, Decay of solutions of the wave equation with a local nonlinear dissipation, Mathematische Annalen 305, 403-417 (1996)

[19] K. Ono, A stretched string equation with a boundary dissipation, Kyushu J. of Math. 28, 265-281 (1994)

[20] J. P. Puel and M. Tucsnak, Boundary stabilization for the von Kármán equation, SIAM J. Control and Optimization 33, 255-273 (1995)

[21] J. P. Puel and M. Tucsnak, Global existence for the full von Kármán system, Applied Mathematics and Optimization 34, 139-160 (1996)

[22] J. Ralston, Solution of the wave equation with localized energy, Comm. Pure Appl. Math. 22, 807-823 (1969)

[23] J. Ralston Gaussian beams and propagation of singularities, Studies in Partial Differential Equations, MAA Studies in Math. 23, W. Littmann, ed., Math. Assoc. America, Washington, D.C., 1982, pp. 206-248

[24] M. Renardy, On the type of certain $C_{0}$-semigroups, Communications in Partial Differential Equations 18, 1299-1307 (1993)

[25] M. Renardy, W. J. Hrusa, and J. A. Nohel, Mathematical Problems in Viscoelasticity, Pitman Monographs and Surveys in Pure and Appl. Math. 35, Longman Sci. Tech., 1987

[26] Wei Xi Shen and Song Mu Zheng, Global smooth solutions to the system of one-dimensional thermoelasticity with dissipation boundary conditions, Chinese Ann. of Math. Ser. B 7, 303-317 (1986)

[27] E. Zuazua, Exponential decay for the semilinear wave equation with locally distributed damping, Communications in Partial Differential Equations 15, 205-235 (1990)

[28] E. Zuazua, Uniform stabilization of the wave equation by nonlinear wave equation boundary feedback, SIAM J. Control and Optimization 28, 466-477 (1990) 